\title{
Evaluation of the internal fixation effect of nano- calcium-deficient hydroxyapatite/poly-amino acid composite screws for intraarticular fractures in rabbits
}

This article was published in the following Dove Press journal:

International Journal of Nanomedicine

\section{Zhenyu Dai ${ }^{1, *}$ \\ Yue $\mathrm{Li}^{2}$,* \\ Yonggang Yan $^{3}$ \\ Ruijie Wan' \\ Qiang Ran' \\ Weizhong Lu' \\ Bo Qiao ${ }^{4}$ \\ Hong $\mathrm{Li}^{3}$ \\ 'Department of Orthopedics, Chongqing Traditional Chinese Medicine Hospital, Chongqing, People's Republic of China; \\ ${ }^{2}$ Department of Clinical Laboratory, the Second Affiliated Hospital, Chongqing Medical University, Chongqing, People's Republic of China; ${ }^{3}$ College of Physical Science and Technology, Sichuan University, Chengdu, People's Republic of China ${ }^{4}$ Department of Orthopedics, the First Affiliated Hospital, Chongqing Medical University, Chongqing, \\ People's Republic of China \\ *These authors contributed equally to this work}

Correspondence: Zhenyu Dai Department of Orthopedics, Chongqing Traditional Chinese Medicine Hospital, No.6 Panxi Seventh Branch Road, Jiangbei District, Chongqing 40002I, People's Republic of China

Email zhenyudail234@I63.com

Hong Li

College of Physical Science and Technology, Sichuan University, Chengdu

Tel +86028 85I 27592

$\mathrm{Fax}+8602885187573$

Email56443848I@qq.com
Tel/fax +860236798 3672 610064, People's Republic of China

Objective: To evaluate the internal fixation effect of nano-calcium-deficient hydroxyapatite/ poly-amino acid (n-CDHA/PAA) composite screws in the intraarticular fracture model.

Materials and methods: A total of $35 \mathrm{New}$ Zealand White rabbits were used in a bilateral femoral intercondylar fracture model and randomly divided into two groups. n-CDHA/PAA screws were used in the experimental group, and medical metal screws were used in the control group. The fracture condition, range of motion, and the screw push-out strength were assessed, and an arthroscopic examination of knee joint was performed at 4, 8, and 12 weeks after surgery. The biodegradation of the n-CDHA/PAA screws in vivo was tested through weighing, and changes in screw structure were assessed by X-ray diffraction at 12 weeks after surgery.

Results: The general situation of all animals was good and showed no incision infection and dehiscence after surgery. X-ray scanning showed that significant callus growth was present in both groups at 4 weeks after surgery, and there was no significant difference $(P>0.05)$ in the Lane-Sandhu score between the experimental and control groups at all time points after surgery. There were no statistically significant differences $(P>0.05)$ in the range of motion and Oswestry Arthroscopy Score of arthroscopic examination of the knee joints between the two groups. The screw push-out strength of the control group was stronger than that of the experimental group at 4 weeks after surgery $(P<0.05)$, but after that, there was no significant difference between the groups $(P>0.05)$. The degradation tests showed that the n-CDHA/PAA screws degraded gradually after implantation, and the weight loss rate was approximately $16 \%$ at 12 weeks after surgery. The X-ray diffraction results showed that the crystal structure of the outer surface of the n-CDHA/PAA screw has changed at 12 weeks after surgery.

Conclusion: The n-CDHA/PAA screw is an effective and safe implant as a potential internal fixation device for an intercondylar fracture of the femur, and its internal fixation effect was similar to that of medical metal screw.

Keywords: n-CDHA/PAA screw, internal fixation, intraarticular fracture, biocompatibility, bioactivity

\section{Introduction}

Currently, the plates, screws, and Kirschner wires used in internal fixation implants are commonly made using stainless steel, titanium, and cobalt alloys. These components have been widely used in orthopedic and trauma surgery because of their mechanical properties and stability. ${ }^{1-3}$ However, there are significant differences between the structure and properties of metal implants and those of bone tissue. Metal implants have many disadvantages such as erosion, allogenic response, bone atrophy, 
MRI interference, and requirements for removal surgery. ${ }^{4-7}$ Using a bioabsorbable implant is one approach to eliminate the need for implant removal after fracture fixation. The ideal bioabsorbable implants can be completely resorbed after surgery. Therefore, secondary surgery to remove implants and long-term interference with the growing skeleton can be prevented. In addition, the risk of implant-associated stress shielding, peri-implant osteoporosis, and infections is reduced. Furthermore, bioabsorbable implants do not interfere with clinical imaging. ${ }^{8}$ Bioabsorbable fixation devices could potentially eliminate these drawbacks associated with metal implants.

The ideal bioabsorbable implant should provide a solid anchorage during the early stages of treatment, followed by a slow biodegradation with substitution by host tissue, and not evoke an immunological or inflammatory response. ${ }^{9-11}$ Absorbable and bioactive implants have attracted major interest and have been used in increasing numbers as an alternative method to metal implants in the orthopedic field during the past decade. To date, many studies have reported the experimental and clinical application of bioabsorbable implants, such as those containing polyglycolic acid, polyL-lactic acid and their copolymers in orthopedic and oral maxillofacial surgery. ${ }^{12-19}$ However, most of them exhibit two main disadvantages. They include relatively low mechanical strength, which can cause intra- and postoperative screw breakage and screw migration, and the complications, including the foreign body reactions, granuloma formation, and allergic reactions induced by degradation products.

To address these issues, a nano-calcium-deficient hydroxyapatite/poly-amino acid (n-CDHA/PAA) composite was designed in the current laboratory and produced through in situ polymerization. Previous studies have shown that n-CDHA/PAA has good biocompatibility, biodegradability, and biomechanics both in vitro and in vivo. ${ }^{20-22}$ It was confirmed that the compressive strength of the n-CDHA/ PAA composites ranges from 88 to $129 \mathrm{MPa}$, which is close to that of natural cortical bone tissue (approximately 50-140 MPa). ${ }^{23}$ This may make it difficult to use n-CDHA/ PAA to maintain the stability of strong diaphyseal fractures, such as those of the femoral shaft or humeral shaft, but may mean that the composites are suitable for non-weight-bearing fractures or low-weight-bearing fractures, such as distal upper limb fractures or some intraarticular fractures. Therefore, in the current study, n-CDHA/PAA composites were fabricated into internal fixation screws, and their internal fixation effect was verified in an intraarticular fracture animal model.

\section{Materials and methods Materials}

The $\varphi 3.5 \times(20-22) \mathrm{mm}$ n-CDHA/PAA screws were provided by Sichuan International Nano Co., Ltd. (Sichuan, People's Republic of China), and the $\varphi 3.5 \times(20-22) \mathrm{mm}$ stainlesssteel screws were purchased from the Wuhan DegeBaier Co., Ltd. (Wuhan, People's Republic of China; Figure 1). The n-CDHA/PAA composites were typically prepared by in situ polymerization. ${ }^{24}$ In brief, the PAA and n-CDHA slurry were added to a three-necked flask with continuous stirring. The reaction system was heated using an oil bath. The mixture was heated to $200^{\circ} \mathrm{C}$ for approximately 2 hours until the water was fully evaporated and the mixture was melted. The system was then kept at $21^{\circ} \mathrm{C}$ for 2 hours and then at $220^{\circ} \mathrm{C}$ for 1 hour. After cooling to room temperature, the target composites were obtained. The reaction system
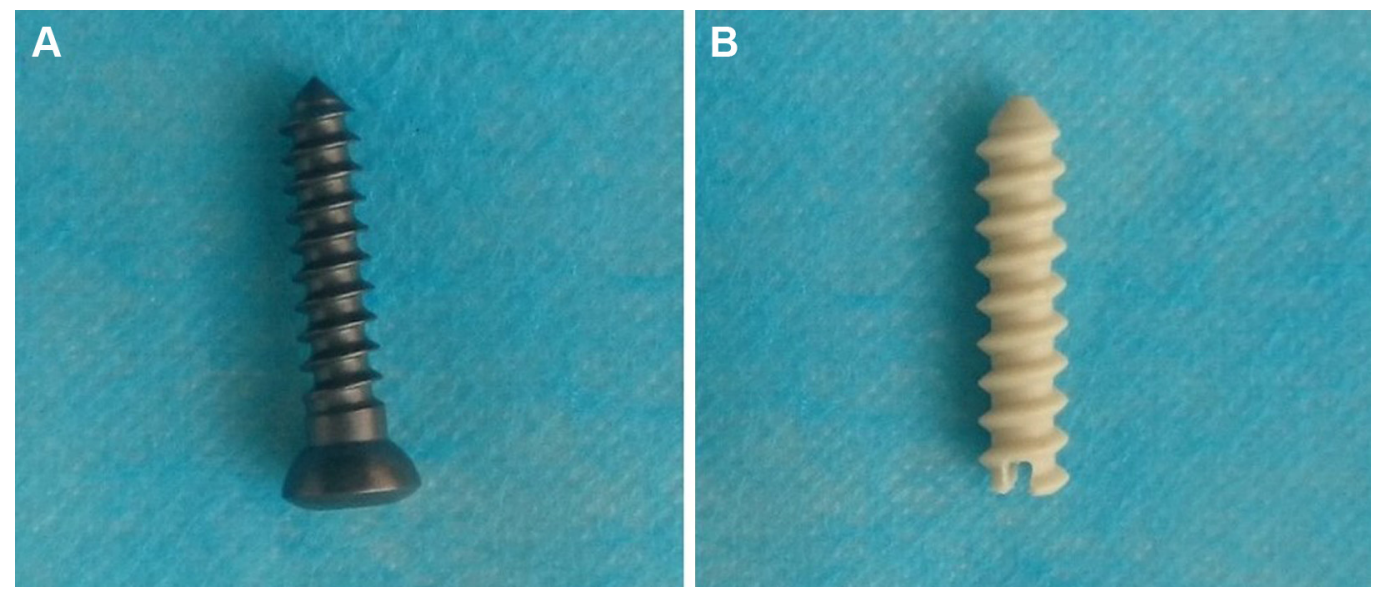

Figure I (A) The $\varphi$ 3.5 $\times(20-22) \mathrm{mm}$ stainless-steel screw and (B) n-CDHA/PAA screw. Abbreviation: $n$-CDHA/PAA, nano-calcium-deficient hydroxyapatite/poly-amino acid. 
was protected by a continuous flow of nitrogen gas to avoid undesirable oxidation reactions. The n-CDHA mass fraction of $\mathrm{n}-\mathrm{CDHA} / \mathrm{PAA}$ was $30 \mathrm{wt} \%$, and the PAA contains six kinds of amino acids: 6-aminocaproic acid, glycine, L-alanine, L-phenylalanine, L-proline, and L-lysine. ${ }^{20}$ All engineered implants were sterilized using the ethylene oxide (EO). The parameters of EO were as follows: sterilization temperature, $56^{\circ} \mathrm{C}$; humidity, $40 \%-60 \%$; EO concentration, $600 \mathrm{mg} / \mathrm{L}$; and sterilization time: 3 hours.

\section{Experimental animals and groups}

Thirty-five mature New Zealand White rabbits, provided by the Laboratory Animal Center, Chongqing Medical University (Chongqing, People's Republic of China), with a mean weight $2.5 \pm 0.5 \mathrm{~kg}$ were used in this study. Thirty animals were randomly divided into the n-CDHA/PAA screw experimental group and the metal screw control group equally. The other five animals were only used to test the in vivo degradation and X-ray diffraction (XRD) analysis of the n-CDHA/PAA screws. Each group was further divided into three subgroups based on the time of analysis of 4, 8, or 12 weeks.

Animals were euthanized after embolism at the specified time points. All animal experiments were approved by the Animal Experimental Committee of Chongqing Medical University (IACUC Number 2016-059), and all procedures were performed in accordance with the National Institutes of Health Guidelines for the Care and Use of Laboratory Animals.

All the rabbits were raised in standard single cages with appropriate bedding, constant temperature $\left(21^{\circ} \mathrm{C} \pm 1^{\circ} \mathrm{C}\right)$, and fed ad libitum. They were anesthetized using 3\% sodium pentobarbital $(1 \mathrm{~mL} / \mathrm{kg})$ intravenously via the ear margin. The animals were fixed in a supine position. All skin preparation, disinfection, draping, and surgical procedures were strictly in accordance with the principle of aseptic surgery. ${ }^{25}$ A conventional $5 \mathrm{~cm}$ longitudinal lateral patellar incision was made, followed by separation of the subcutaneous tissue, fascia, and muscle to expose the patella. The patella was dislocated to expose the femoral condyle. Then, an intercondylar fracture of the femur was created using a blade saw (Figure 2A). After temporary reduction, one hole was drilled outward from the lateral side to the contralateral cortex using a $2.5 \mathrm{~mm}$ diameter drill. The experimental group was fixed using n-CDHA/PAA screws, and the control group was fixed on the opposite side using metal screws (Figure 2B and C), followed by suturing and topical skin closure, disinfection, and application of erythromycin ointment to the incisions. Each animal received intramuscular injection of penicillin (2,00,000 units daily) for the first 3 days after the surgery.

All animals with fractures were monitored daily postoperatively for signs of abnormal mental behavior, significant limping, incision infection, food intake, and screw exposure.

Additionally, the fixed fractures were visually assessed at 4,8 , and 12 weeks after surgery.

\section{Radiological analysis after surgery}

$\mathrm{X}$-ray scanning was performed to evaluate fracture healing at 4,8 , and 12 weeks after surgery. The X-ray parameters included $52 \mathrm{kV}$ voltage, a course of 1 second, current of $100 \mathrm{~mA}$, and a distance between the ball tube and sample of $80 \mathrm{~cm}$. The Lane-Sandhu scoring system was applied for radiographic outcomes and bone healing in the two groups. ${ }^{26}$ Radiographic scores were compared between the two groups. All radiographic results were evaluated in randomized and double-blind conditions.
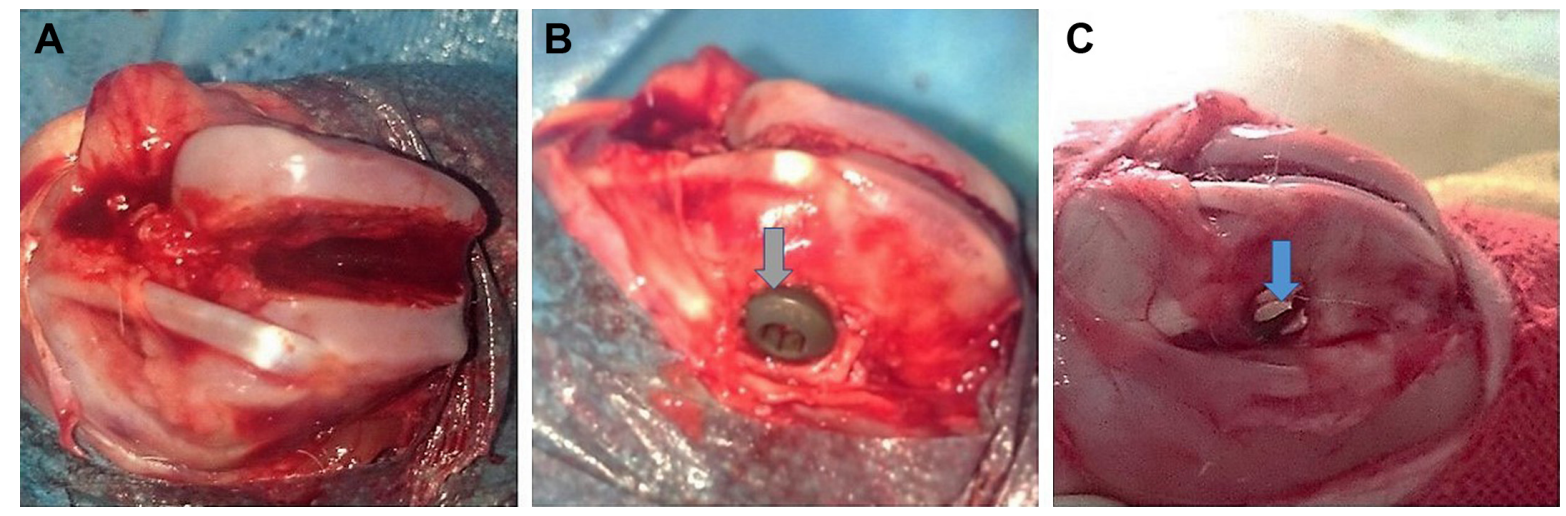

Figure 2 (A) The intercondylar fracture model of the femur; (B) control group was fixed with metal screw; and (C) experimental group was fixed with n-CDHA/PAA screw. Note: Gray arrow indicates the metal screw cap and blue arrow indicates the n-CDHA/PAA screw cap.

Abbreviation: n-CDHA/PAA, nano-calcium-deficient hydroxyapatite/poly-amino acid. 


\section{Range of motion of the knee joint}

Range of motion (or ROM) is the linear or angular distance that a moving object may normally travel while properly attached to another. It is also called range of travel, particularly when talking about mechanical devices and in mechanical engineering fields. The ROM of the knee joint was measured at the predetermined time points. A specific method has been previously reported. ${ }^{27,28}$ Briefly, three points, including the proximal femur, distal tibia, and lateral femoral condyle, were assessed using a plastic goniometer to measure the knee joint angle. The lateral femoral condyle was the central fulcrum of the goniometer. The arms of the goniometer were aligned with the proximal femur and distal tibia. All angles were measured relative to the neutral $0^{\circ}$ axis during extension of the femur shaft. Each animal was measured blindly three times at each time point and the average value was calculated.

\section{Arthroscopic assessment}

An arthroscopic assessment was performed at 4, 8, and 12 weeks after surgery. The results were evaluated using the adjusted Oswestry Arthroscopy Score (OAS) system, which has been validated for qualitative repair assessment in humans, and is based on the arthroscopic visualization of hyaline articular cartilage..$^{29,30}$ This score ranges from 0 to 10 points (Table 1), with higher values indicating a better quality of fracture healing. The observer visually assessed the fracture gap level with the surrounding cartilage (0-2), integration of

Table I The adjusted OAS system

\begin{tabular}{l|l}
\hline OAS & Points \\
\hline Fracture gap level with surrounding cartilage & 2 \\
Level & 1 \\
Raised & 0 \\
Below & \\
Integration of fracture gap level with & \\
surrounding cartilage & 2 \\
Complete & 1 \\
Minor disruption ( $<25 \%$ of area) & 0 \\
Major disruption ( $>25 \%$ of area) & \\
Stiffness in fracture gap & 2 \\
Normal compared with adjacent cartilage & 1 \\
Softer & 0 \\
Very soft/hard & \\
Appearance of articular surface & 2 \\
Smooth & 1 \\
Fine fronds & 0 \\
Severe fronds/fibrillation & \\
Color of articular surface & \\
Pearly, hyaline-like & 2 \\
White & 1 \\
Yellow bone & 0 \\
Total & $0-10$ \\
\hline Abbreviaton: OAS, Oswsty Arthrossopy Score
\end{tabular}

Abbreviation: OAS, Oswestry Arthroscopy Score. fracture gap level with the surrounding cartilage (0-2), stiffness in the fracture gap (0-2), the appearance of the articular surface $(0-2)$, and the color of the articular surface $(0-2){ }^{30}$ The blinded measurements were performed three times at each time point, and the average value was calculated.

\section{Axial push-out strength test}

The rabbits were sacrificed at 4,8 , and 12 weeks after surgery. The distal femoral condyles were harvested after the surrounding soft tissues were dissected. The axial push-out strength of the screws was tested using a QJ212C electronic universal testing machine (Shanghai QingJi Instrument Co., Ltd, Shanghai, People's Republic of China; Figure 3). The harvested distal femoral condyle was embedded in methyl methacrylate cement (Figure 3B), aligning the long axis of the screw with the true axial pushout, and clamped in the testing machine. The cement was placed in two clamped metal holders (Figure 3C) and the head of screw clamped vertically. The cement block remained static while the screw was pushed out by a push bin (Figure 3A) at a speed of $0.1 \mathrm{~mm} / \mathrm{second}$ using an axial tensile testing mode. The load value was recorded when the implant began to loosen.

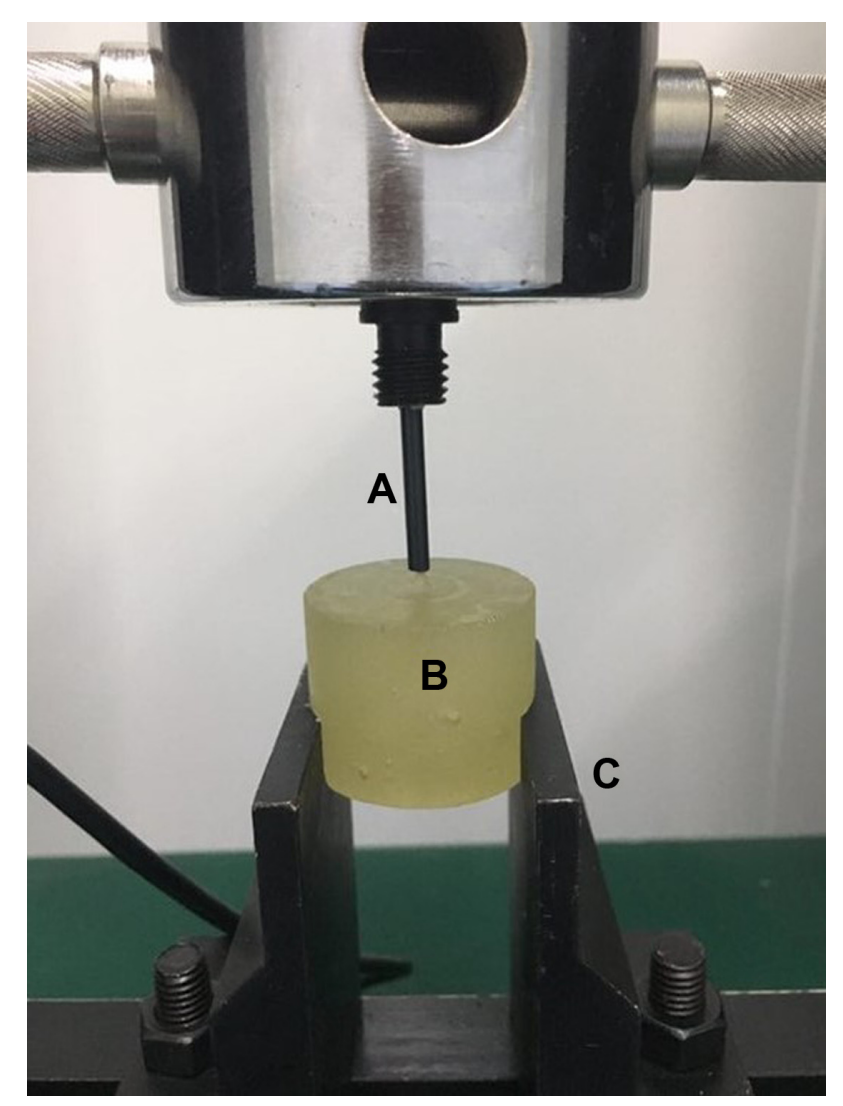

Figure 3 The axial push-out test diagram.

Notes: (A) Push bin; (B) methyl methacrylate cement (including the harvested distal femoral condyle); and (C) clamp. 
The standard for determining screw loosening was that the screw displacement increases but the loading strength does not increase. ${ }^{31}$ Each group was tested at least three times.

\section{In vivo degradation of the $n-C D H A / P A A$ screws}

The weight loss rate of the n-CDHA/PAA screw was evaluated at 4,8 , and 12 weeks after surgery. The n-CDHA/PAA screws were withdrawn after removing the adhered tissues, and then were ultrasonically rinsed with $180 \mathrm{~g} / \mathrm{L}$ chromic acid and $10 \mathrm{~g} / \mathrm{L}_{\mathrm{AgNO}_{3}}$ solution followed by distilled water and dried under vacuum at $30^{\circ} \mathrm{C}$ for 24 hours. The samples were weighed, and the weight loss rate was calculated. ${ }^{32,33}$ Five samples from each were tested. The weight loss rate (WLR) was calculated as follows:

$$
\mathrm{WLR}=\frac{M_{0}-M_{1}}{M_{0}} \times 100 \%
$$

where $M_{0}$ is the original weight $(\mathrm{g})$ and $M_{1}$ is the weight after implantation. XRD was performed to analyze possible changes in the crystal structure of the n-CDHA/PAA screw surface at 12 weeks after surgery, including its inside and outer surfaces.

\section{Statistical analysis}

Statistical analysis was performed using SPSS 19.0 (SPSS Inc., Chicago, IL, USA) statistical software. The measurement data are presented as mean \pm standard deviation (SD).
The data were analyzed separately in accordance with the time point and experimental/control group using one-way ANOVA with post hoc Tukey's tests. Also $t$-tests were carried out in the scoring system results to determine differences between the two material groups at each time point. $P<0.05$ was considered to indicate statistical significance.

\section{Results \\ General observation}

All the animals showed no obvious mental abnormalities, loss of appetite, incision infection, or screw exposure after surgery. Lameness was found in both groups at 4 weeks after surgery, but this gradually disappeared by 12 weeks after surgery. The collected distal femoral condyles, embraced metal or n-CDHA/PAA screws, showed no obvious inflammatory reactions, such as swelling or fluid accumulation, necrosis, and osteolysis, and no bleaching of the material was observed in the peripheral bone tissues at all time points after surgery. The fracture line was still observed, but there was an obvious bony connection in both groups at 4 weeks after surgery. A distinct thin layer of new bone tissue could be found in the fracture gaps of all animals at 8 weeks after surgery. The screw caps of the n-CDHA/PAA group were covered by a layer of new bone tissue at 12 weeks after surgery, but only fibrous tissue was observed in the control group (Figure 4A-F).

\section{Radiological analysis after surgery}

The results of X-ray scanning of the knee joint are shown in Figure $5 \mathrm{~A}-\mathrm{C}$. There were similar results at all time points
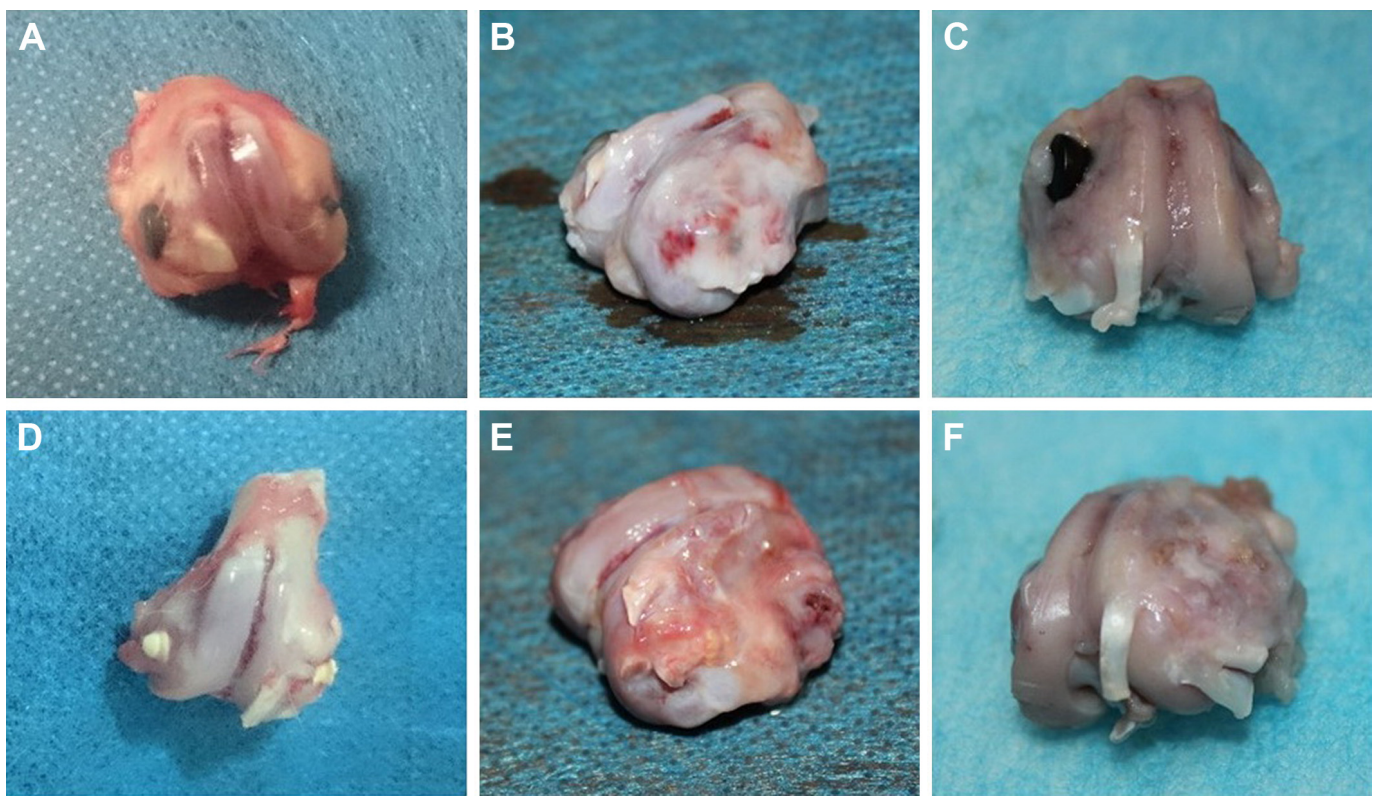

Figure 4 The specimen of harvested distal femoral condyles at 4 (A), 8 (B), and 12 (C) weeks after surgery in the control group; the specimen of harvested distal femoral condyles at $4(\mathbf{D}), 8(\mathbf{E})$, and $12(\mathbf{F})$ weeks after surgery in the experimental group. 
A a
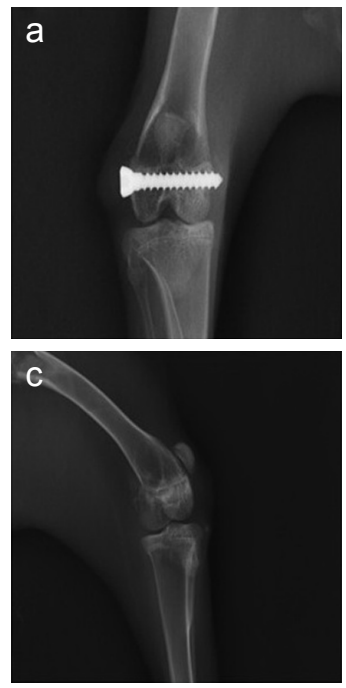

B
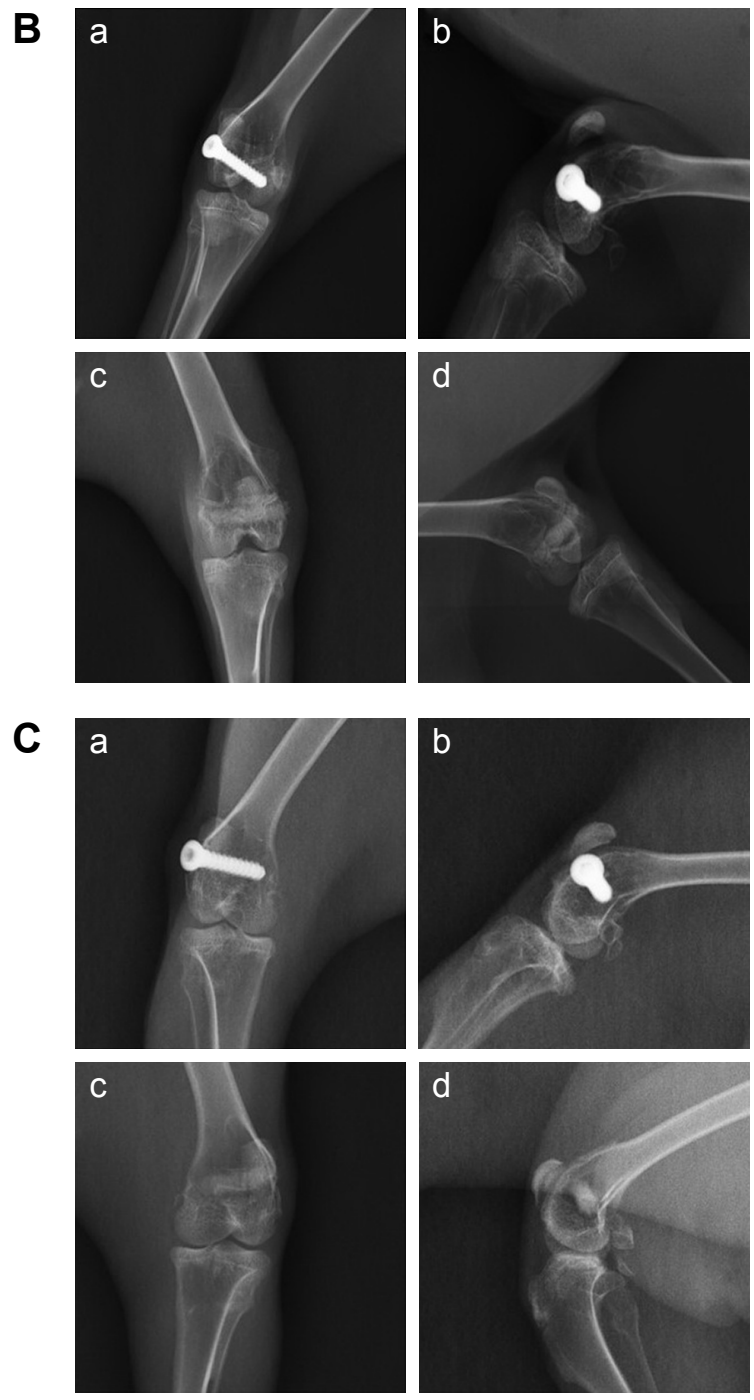

Figure 5 (A) The $\mathrm{X}$-ray results at 4 weeks after surgery in the control group (a, anteroposterior view; b, lateral view) and in the experimental group (c, anteroposterior view; d, lateral view). (B) The X-ray results at 8 weeks after surgery in the control group (a, anteroposterior view; b, lateral view) and in the experimental group (c, anteroposterior view; d, lateral view). (C) The X-ray results at 12 weeks after surgery in the control group (a, anteroposterior view; b, lateral view) and in the experimental group (c, anteroposterior view; d, lateral view). in both groups. At 4 weeks after surgery, the X-ray scanning showed that there was a significant callus growth in both groups. The fracture lines became blurred, and the fracture gap did not widen. The fracture line gradually disappeared by 8 weeks after surgery, and the fracture had healed completely by 12 weeks after surgery. During the whole experimental period, no screw breakage and loosening were observed, and no obvious bone destruction or bone mineral density abnormalities were observed in the surrounding bone tissue. According to the Lane-Sandhu scores, no statistically significant difference $(P>0.05)$ was found between the two groups (Figure 6). The results of X-ray scanning were consistent with those of the Lane-Sandhu score.

\section{ROM of the knee joint}

The ROM can reflect the functional recovery of the knee joint. The results of the ROM test are presented in Figure 7. The ROM in both groups gradually increased after surgery. The mean values of the experimental and control groups were $51.55^{\circ} \pm 3.30^{\circ}$ and $50.38^{\circ} \pm 3.31^{\circ}$, respectively, at 4 weeks after surgery, probably because of lack of functional exercise and early joint adhesion. However, a significant recovery in ROM was found at 12 weeks after surgery, and it was $122.05^{\circ} \pm 5.24^{\circ}$ and $121.98^{\circ} \pm 5.08^{\circ}$ for the experimental and control groups, respectively. There was no significant difference $(P>0.05)$ between the two groups at the different time points, but the ROM results observed in the study are lower than the normal ROM of a rabbit. ${ }^{34}$

\section{Arthroscopic assessment}

The semiquantitative OAS results, reflecting a macroscopic assessment of harvested distal femoral condyles, are shown in Table 2, and there was a similar result in the two groups. Compared with the n-CDHA/PAA group, the fracture gap level with surrounding cartilage and the integration of the fracture gap level with surrounding cartilage were slightly better in the control group, but the color and appearance of the articular surface in the n-CDHA/PAA group were better than those of the metal screw group. This suggested that the n-CDHA/PAA screws might have better biocompatibility than the metal screws.

\section{Axial push-out test}

The results of the push-out strength test are shown in Figure 8. At 4 weeks after surgery, the push-out strength of the n-CDHA/PAA screw was $231 \pm 19.48 \mathrm{~N}$, and that of the metal screw was $272.0 \pm 8.6 \mathrm{~N}$. There was a statistically significant difference $(P<0.01)$ between the two groups. At 8 and 12 weeks after surgery, the push-out strength of the 


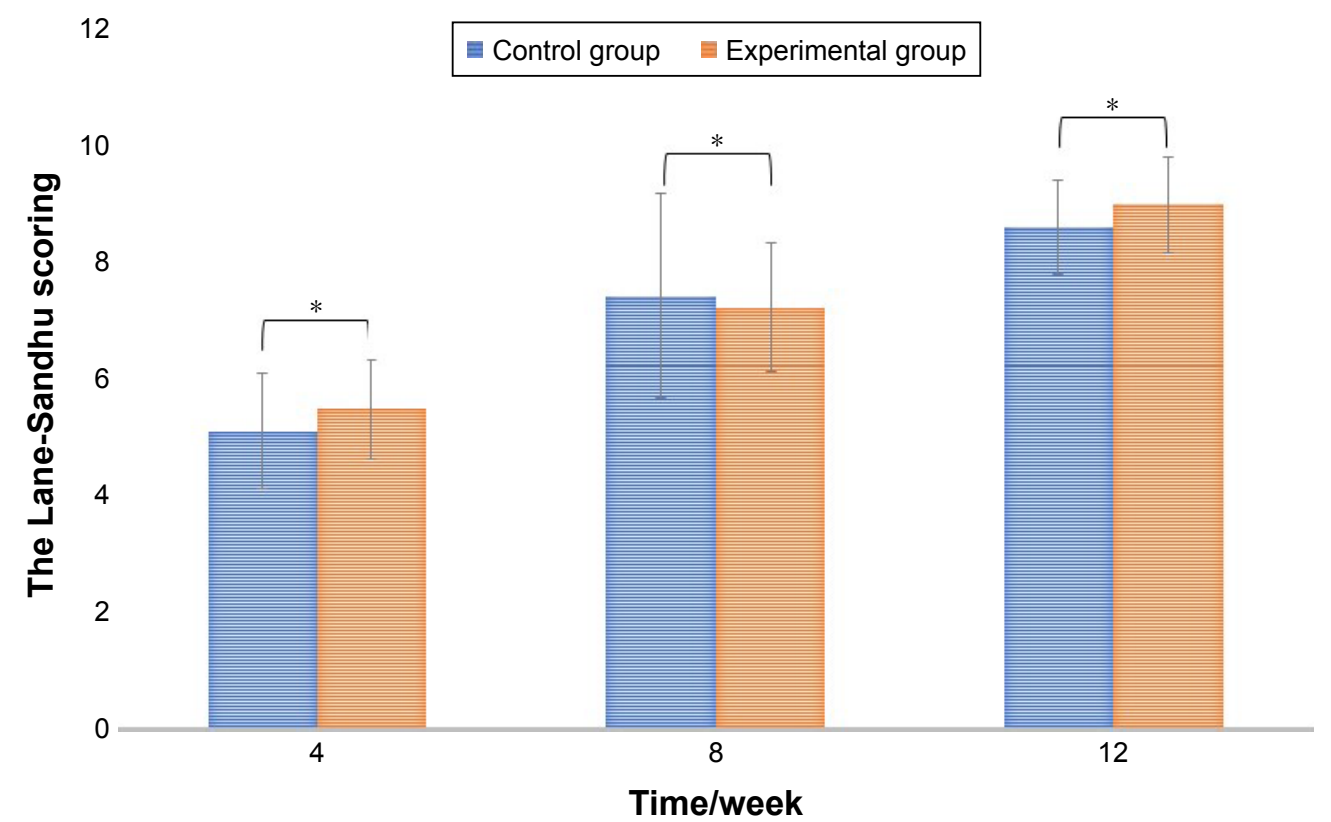

Figure 6 The Lane-Sandhu scores in the control and experimental groups. Note: $* P>0.05$, compared with control group $(n=5)$.

n-CDHA/PAA screw was still lower than that of the metal screw, but there was no statistically significant difference $(P>0.05)$ between them.

\section{In vivo degradation}

Figure 9 shows the weight loss of the n-CDHA/PAA screw. There was a reduction of approximately $5.0 \%$ in weight for the n-CDHA/PAA screw at 4 weeks after surgery, and
$16 \%$ remained at 12 weeks after surgery. The XRD analysis before and after implantation of the n-CDHA/PAA screw is shown in Figure 10. The characteristic peak of the outer surface of $n-C D H A$ component at $2 \theta=20^{\circ}$ before implantation was lower than that after implantation. Compared with outer surface results, the characteristic peaks of the internal cross-section of the n-CDHA and PAA components were unchanged before and after surgery.

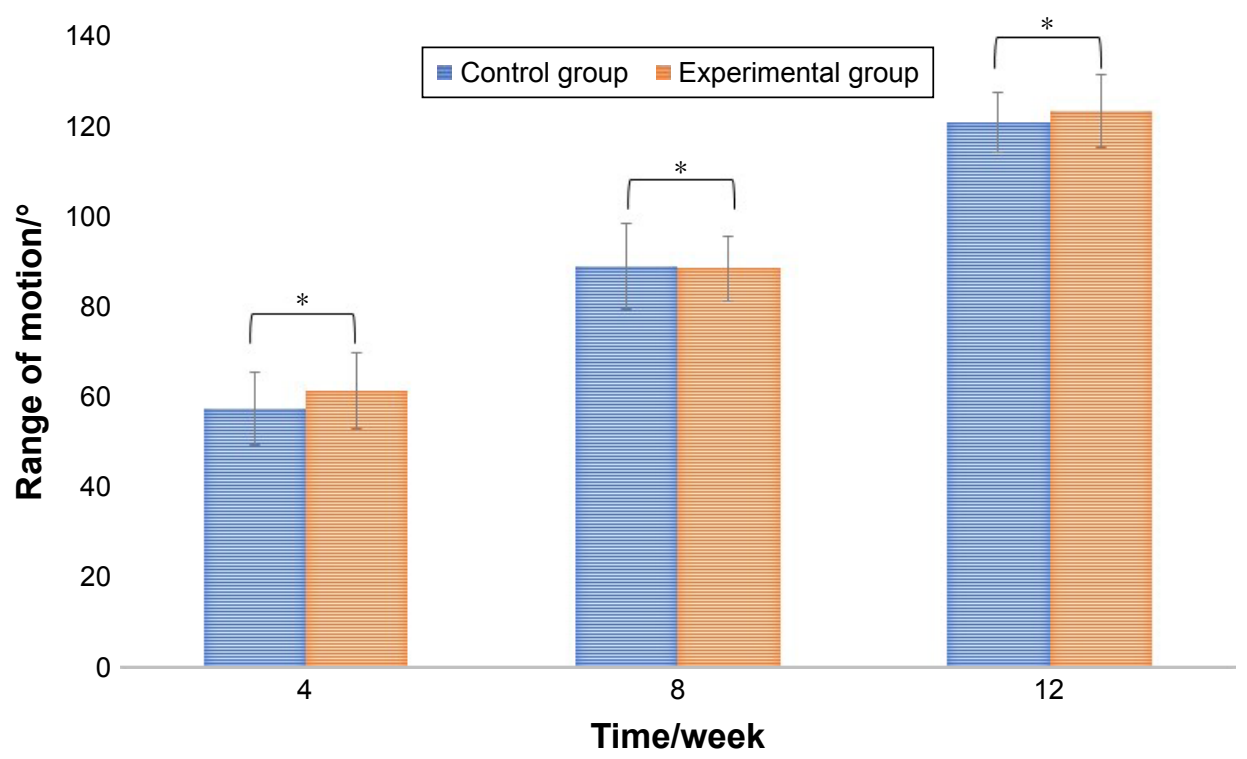

Figure 7 The range of motion of knee joints in the control and experimental groups. Note: ${ }^{*} P>0.05$, compared with control group $(n=5)$. 
Table 2 The results of adjusted OAS for femoral intercondylar fracture

\begin{tabular}{|c|c|c|c|c|c|c|c|c|c|c|c|c|c|c|c|c|c|}
\hline \multirow[t]{2}{*}{$\begin{array}{l}\text { Time } \\
\text { (weeks) }\end{array}$} & \multirow[t]{2}{*}{ Group } & \multicolumn{3}{|c|}{$\begin{array}{l}\text { Appearance of } \\
\text { articular surface }\end{array}$} & \multicolumn{3}{|c|}{$\begin{array}{l}\text { Color of } \\
\text { articular surface }\end{array}$} & \multicolumn{3}{|c|}{$\begin{array}{l}\text { Fracture gap } \\
\text { level with } \\
\text { surrounding } \\
\text { cartilage }\end{array}$} & \multicolumn{3}{|c|}{$\begin{array}{l}\text { Integration of } \\
\text { fracture gap } \\
\text { with surrounding } \\
\text { cartilage }\end{array}$} & \multicolumn{3}{|c|}{$\begin{array}{l}\text { Stiffness in } \\
\text { fracture gap }\end{array}$} & \multirow[t]{2}{*}{ Total } \\
\hline & & Min & Max & Avg & Min & Max & Avg & Min & Max & Avg & Min & Max & Avg & Min & Max & Avg & \\
\hline \multirow[t]{2}{*}{4} & Control & 0 & 1 & 0.6 & 0 & 1 & 0.6 & 0 & 1 & 0.6 & 0 & 1 & 0.6 & 0 & 1 & 0.8 & 3.2 \\
\hline & Experimental & 0 & 2 & 0.8 & 0 & 2 & 1.0 & 0 & 1 & 0.4 & 0 & 1 & 0.6 & 0 & 1 & 0.8 & 3.6 \\
\hline \multirow[t]{2}{*}{8} & Control & 0 & 1 & 0.8 & 1 & 2 & 1.2 & 0 & 2 & 1.2 & 1 & 2 & 1.4 & 1 & 2 & 1.4 & 6.0 \\
\hline & Experimental & 0 & 2 & 1.2 & 1 & 2 & 1.4 & 0 & 2 & 1.0 & 1 & 2 & 1.2 & 1 & 2 & 1.2 & 6.0 \\
\hline \multirow[t]{2}{*}{12} & Control & 1 & 2 & 1.4 & 1 & 2 & 1.6 & 1 & 2 & 1.8 & 1 & 2 & 1.8 & 1 & 2 & 1.8 & 8.4 \\
\hline & Experimental & I & 2 & 1.8 & I & 2 & 1.8 & I & 2 & 1.6 & I & 2 & 1.6 & I & 2 & 1.8 & 8.6 \\
\hline
\end{tabular}

Abbreviation: OAS, Oswestry Arthroscopy Score.

\section{Discussion}

Intraarticular fractures are one of the most common clinical fractures. These fractures can damage the articular cartilage and the integrity of the articular surface, and so even simple fractures often require surgical fixation to reduce the disability rate after injury. Strong internal fixation of osteochondral fragments contributes to early recovery of joint activity and acceptance of physiotherapy to avoid joint adhesion and reduce the incidence of posttraumatic arthritis. For simple intraarticular fractures, metal screws are often used clinically, rather than screw-plate systems. ${ }^{35}$ Metal screws demonstrate biological inertia and high strength. Their biological inertia can reduce their stimulation after implantation in vivo, and the high strength ensures a stable fixation. However, metal screws are prone to breakage after long-term implantation because of their fatigability, and the biological inertia easily leads to screw withdrawal because of the non-bony connection with the surrounding bone tissue. Furthermore, the destruction of surrounding bone tissue or intraarticular infection caused by the prolonged and slow release of metal ions has also been reported in some clinical cases. ${ }^{36,37}$ Therefore, in view of the characteristics of n-CDHA/PAA composites, we used them to make biomimetic screws, and explored their potential feasibility for the internal fixation of simple intraarticular fractures.

Good biocompatibility is one of the basic requirements for clinical application of a biomaterial. It has been regulated by different set of rules globally such as the Food and Drug Administration and the International Standards Organization (ISO). ${ }^{37-39}$ To evaluate the biocompatibility of self-made n-CDHA/PAA composites, we performed the relative in vitro and in vivo tests, in accordance with the ISO10993-1 standards before the current study. The results showed that the novel n-CDHA/PAA composite can promote MG-63 osteoblast-like cell adhesion and proliferation in vitro and it does not cause significant inflammation after implantation

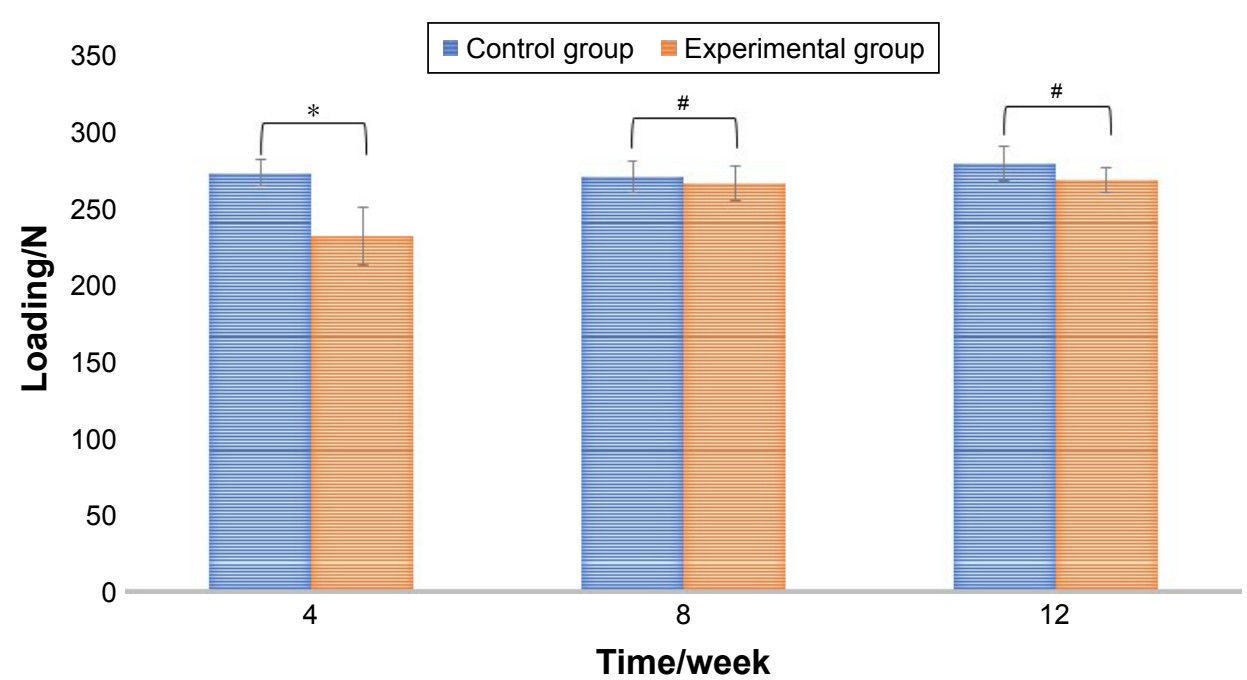

Figure 8 The results of push-out strength in the control and experimental groups. Note: ${ }^{*} P<0.01,{ }^{\#} P>0.05$, compared with control group $(n=5)$. 


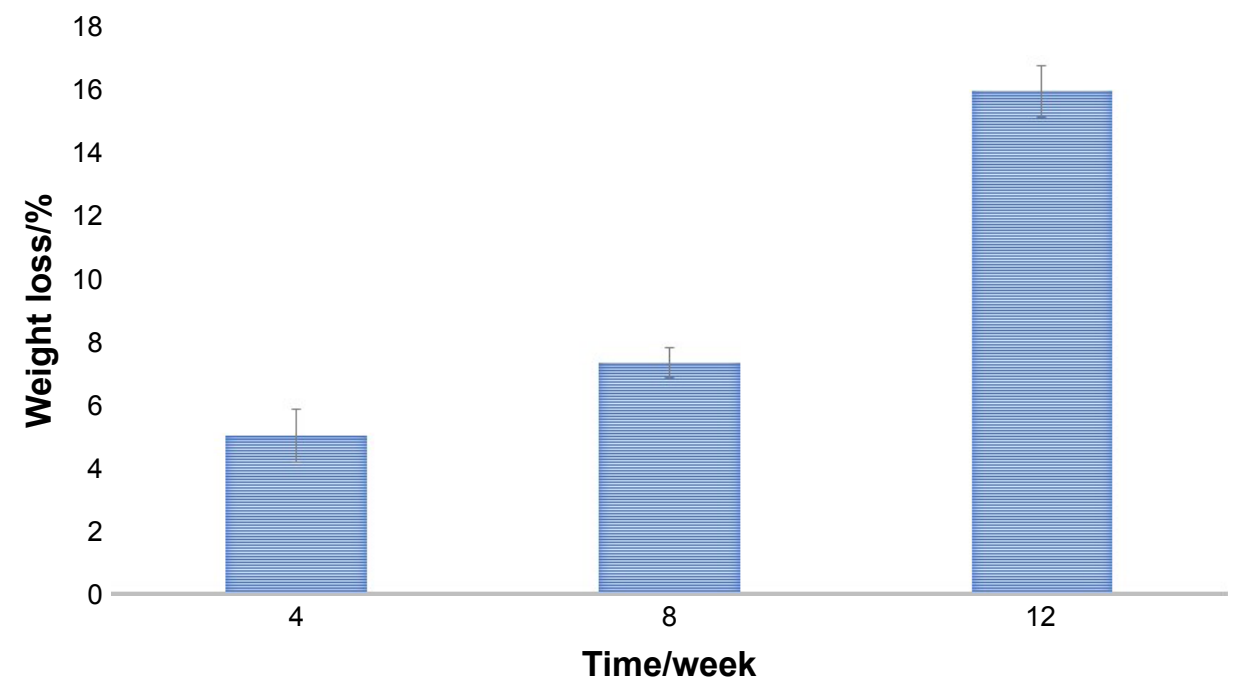

Figure 9 The weight loss of $n-C D H A / P A A$ screw in vivo $(n=5)$.

Abbreviation: n-CDHA/PAA, nano-calcium-deficient hydroxyapatite/poly-amino acid.

in vivo. ${ }^{20,21}$ In the current study, a visual assessment and arthroscopic and X-ray observations performed after surgery indicated that there was no obvious inflammation or other unexpected pathological changes in the experimental group. The results of the current study are consistent with those of our previous research. Additionally, previous studies have shown that n-CDHA and PAA have good biocompatibility, individually. ${ }^{40-42}$ XRD and Fourier infrared also showed that the respective original crystalline status and physical and chemical properties of n-CDHA and PAA components in the n-CDHA/PAA composites did not change. ${ }^{20}$ Therefore, considering the results of present and previous studies, we

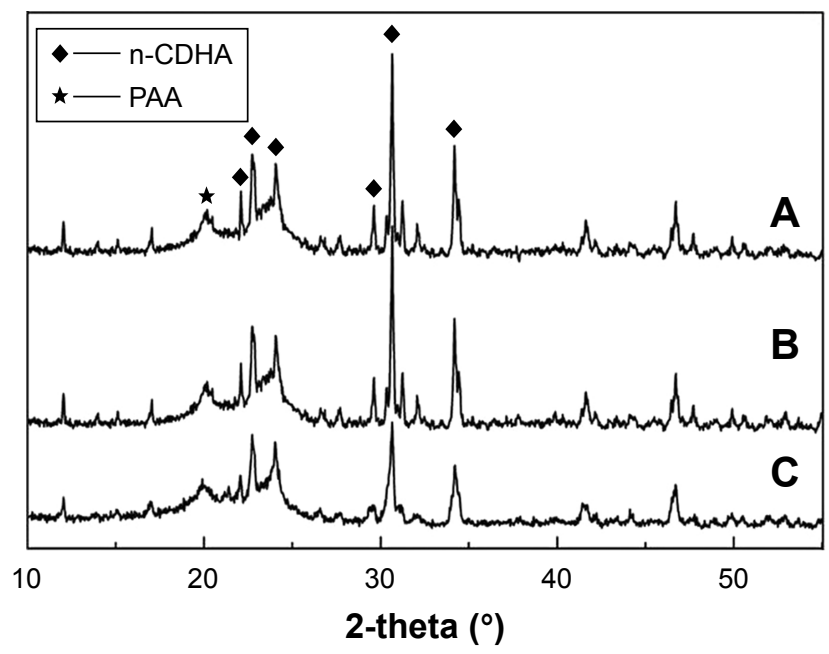

Figure 10 The result of XRD of $n-C D H A / P A A$ screw.

Notes: (A) The outer surface of $n-C D H A / P A A$ before implantation; (B) The internal cross-section of $n-C D H A / P A A$ after implantation; (C) The outer surface of n-CDHA/PAA after implantation.

Abbreviations: n-CDHA/PAA, nano-calcium-deficient hydroxyapatite/poly-amino acid; XRD, X-ray diffraction. believe that the n-CDHA/PAA composite screws have good biocompatibility.

Biodegradability is one of the main advantages of absorbable materials. The degradation of polymeric biomaterials usually includes bulk degradation and surface erosion degradation. Surface erosion degradation refers to the hydrolysis of the side chain or the splitting of the crosslink point to produce a soluble polymer. It occurs on the surface of the material, while the internal structure of the material remains essentially unchanged. Importantly, surface erosion degradation can be conducive to maintaining a good mechanical retention rate during the bone tissue healing. Duan et al reported that n-CDHA/PAA composites are degradable and investigated a surface erosion degradation model as part of an in vitro degradability experiment. ${ }^{43}$ In the current study, the XRD results showed that the characteristic peak of the outer surface of n-CDHA component at $2 \theta=20^{\circ}$ decreased after surgery, but the characteristic peaks of the internal cross-section of the n-CDHA and PAA components were unchanged before and after surgery. This implied that the n-CDHA/PAA screws could be degraded by surface erosion.

In the current study, the weight loss rate of the n-CDHA/ PAA screws in vivo was approximately $16 \%$ at 12 weeks after surgery, which is different compared with that reported in vitro. ${ }^{20}$ Many previous studies have also reported a difference between the in vivo and in vitro degradation rates of biomaterials, which results from significant environmental differences between in vitro and in vivo conditions. ${ }^{44-46}$ Our previous experiments demonstrated that $n-C D H A / P A A$ composites become wrapped in fibrous tissue during the process of muscle implantation, while wrapped in fibrous tissue 
at the early stage and covered by new bone tissue at the later stage during the process of bone implantation. ${ }^{21}$ This indicates that the composites could have different biochemical reactions at different sites of implantation because of different physical and chemical environments in different tissues. Furthermore, we speculate that when the composites become wrapped in tissue after implantation, their contact area with bodily fluids would be decreased, which inevitably would affect the enzymolysis and hydrolysis occurring on and in the implants. These factors might explain why the degradation rates of n-CDHA/PAA composites in vivo and in vitro are completely different. However, the specific mechanism still requires further study.

For internal fixation screws, the strength of stable fixation should be maintained at least until clinical healing of the fracture. ${ }^{47-49}$ Apart from factors that arise during surgery and quality of bone, the internal fixation failure of rigid metal screws is mainly caused by insufficient holding power. The holding power and push-out or pull-out strength represent the maximum recorded tensile strength that is required to pull the screws out of the bone or cause screw failure. ${ }^{50}$ In the current study, the push-out strength of the n-CDHA/PAA screws was significantly lower than that of the metal screws at 4 weeks after surgery, but as time progressed, there was no significant difference in the push-out strength between the two groups. Although the push-out strength data of the n-CDHA/PAA screws at 4 weeks after surgery were not satisfactory, the other results, including the general observation and X-ray scanning results, confirmed that the n-CDHA/ PAA screws suffered no breakage, loosening, or fragment displacement during the whole experimental period. These results show that the strength of the n-CDHA/PAA screws is suitable for the fixation of simple femoral intercondylar fractures. Our previous report of an n-CDHA/PAA bone implantation test showed that bone-bonding was achieved between the n-CDHA/PAA composite and the surrounding bone tissue. ${ }^{21}$ It can be inferred that the strength of bonebonding between the n-CDHA/PAA and bone is weaker than the mechanical bonding between a metal screw and bone tissue in the early stages after surgery. Future research should focus on improving the mechanical bonding strength between nonmetallic screws and bone tissue in the early stages after surgery. Previously reported studies have shown that the performance of screws during bone fixation is affected by different parameters such as thread depth, length, shape, and surface finish. The mechanical properties of screw materials, and the flexural and push-out properties of composite screws can vary with the thread and width of a screw. 7,31,51,52
Therefore, adjusting the physical form of composite screws could be a potential and effective method for improving early fixation strength of such nonmetallic screws.

Postoperative functional outcomes are an important criterion in evaluating fracture treatments. Postsurgical inflammation, soft tissue edema, wound healing, joint adhesion, and contracture represent some of the most challenging issues in ensuring an early recovery after intraarticular surgery. ${ }^{53,54}$ A good postoperative situation usually forebodes satisfactory functional outcomes. ROM and arthroscopic assessment have been recognized as important outcome measures and major components of joint-specific scoring systems after knee joint operations. ${ }^{29,55}$ In the current study, the experimental group displayed similar results to the control group in terms of ROM, and an arthroscopic assessment also revealed that the articular surfaces in the two groups were in a good condition after surgery. The evaluation of ROM and OAS results for knee joint function was cautious and objective in the current study, but it is also important to account for the perception of patients of their treatment outcomes, which includes the absence of pain and a return to daily activities, with the knowledge that these factors can adversely affect the success of postoperative functional recovery and therefore the overall clinical results. ${ }^{56,57}$ Excessive and continuous pain would obviously affect mental behavior, food intake, and joint function. ${ }^{58,59}$ In the current study, the rabbits did not show abnormal mental behavior or loss of appetite in both groups. This indirectly indicates that the n-CDHA/PAA screws did not cause additional and excessive pain compared with the metal screws, and this would contribute to the recovery of postoperative function. Unfortunately, unlike in clinical human trials, we cannot directly comment on these crucial patient perceptions in animal experiments.

In summary, compared with medical metal screws, absorbable n-CDHA/PAA biological screws can avoid the stress shielding effect, screw withdrawal, screw breakage, and magnetic resonance interference during long-term implantation. In addition, they can avoid the removal of an internal fixator. Therefore, they can also avoid the economic costs and surgical risks associated with secondary surgery, such as infection, pain, joint adhesion, and anesthesia risks.

\section{Conclusion}

In the current study, the internal fixation effect of novel self-made n-CDHA/PAA screws was evaluated through an experimental femoral intercondylar fracture model in animals. The results indicate that the novel n-CDHA/PAA screws have good biocompatibility, biodegradability, and 
the internal fixation effect is similar to that of medical metal screws. Within 3 months after surgery, bone-bonding between the n-CDHA/PAA screw and bone tissue was gradually achieved. The surface erosion degradation of the n-CDHA/PAA screw was confirmed by XRD. The n-CDHA/ PAA screw has the potential to be used as an internal fixation device for intraarticular fractures. However, its long-term biocompatibility, biodegradation, and biomechanics in vivo require further investigation.

\section{Acknowledgments}

This work was supported by the National Natural Science Foundation of China (No 51771294) and the plan of young and middle-aged medical high-end reserve talent of Chongqing.

\section{Disclosure}

The authors report no conflicts of interest in this work.

\section{References}

1. Buijs GJ, van der Houwen EB, Stegenga B, Bos RR, Verkerke GJ Torsion strength of biodegradable and titanium screws: a comparison. J Oral Maxillofac Surg. 2007;65(11):2142-2147.

2. Lawson KJ, Brems J. Effect of insertion torque on bone screw pullout strength. Orthopedics. 2001;24(5):451-454.

3. Disegi JA, Eschbach L. Stainless steel in bone surgery. Injury. 2000; 31(Suppl 4):D2-D6.

4. Shellock FG, Mink JH, Curtin S, Friedman MJ. MR imaging and metallic implants for anterior cruciate ligament reconstruction: assessment of ferromagnetism and artifact. J Magn Reson Imaging. 1992;2(2): 225-228.

5. Pihlajamäki H, Kinnunen J, Böstman O. In vivo monitoring of the degradation process of bioresorbable polymeric implants using magnetic resonance imaging. Biomaterials. 1997;18(19):1311-1315.

6. Weiler A, Hoffmann RF, Stähelin AC, Helling HJ, Südkamp NP. Biodegradable implants in sports medicine: the biological base. Arthroscopy. 2000;16(3):305-321.

7. Felfel RM, Ahmed I, Parsons AJ, Rudd CD. Bioresorbable screws reinforced with phosphate glass fibre: manufacturing and mechanical property characterisation. J Mech Behav Biomed Mater. 2013;17: $76-88$.

8. Wu CM, Chen YA, Liao HT, Chen CH, Pan CH, Chen CT. Surgical treatment of isolated zygomatic fracture: outcome comparison between titanium plate and bioabsorbable plate. Asian J Surg. 2018;41(4): 370-376.

9. Jackson DW, Simon TM. Tissue engineering principles in orthopaedic surgery. Clin Orthop Relat Res. 1999;(367 Suppl):S31-S45.

10. Temenoff JS, Mikos AG. Injectable biodegradable materials for orthopedic tissue engineering. Biomaterials. 2000;21(23):2405-2412.

11. Magarelli N, Savastano MA, Palmieri D, et al. Poly-L-lactic acid betatricalcium phosphate screws: a preliminary in vivo biocompatibility study. Int J Immunopathol Pharmacol. 2007;20(1):207-211.

12. Bergsma JE, Rozema FR, Bos RR, Boering G, de Bruijn WC, Pennings AJ. Biocompatibility study of as-polymerized poly(L-lactide) in rats using a cage implant system. J Biomed Mater Res. 1995;29(2): 173-179.

13. Eppley BL, Reilly M. Degradation characteristics of PLLA-PGA bone fixation devices. J Craniofac Surg. 1997;8(2):116-120.
14. Deguchi M, Cheng BC, Sato K, Matsuyama Y, Zdeblick TA. Biomechanical evaluation of translaminar facet joint fixation. A comparative study of poly-L-lactide pins, screws, and pedicle fixation. Spine. 1998; 23(12):1307-1313.

15. Fuchs M, Köster G, Krause T, Merten HA, Schmid A. Degradation of and intraosseous reactions to biodegradable poly-L-lactide screws: a study in minipigs. Arch Orthop Trauma Surg. 1998;118(3):140-144.

16. Bergsma JE, de Bruijn WC, Rozema FR, Bos RR, Boering G. Late degradation tissue response to poly(L-lactide) bone plates and screws. Biomaterials. 1995;16(1):25-31.

17. Jukkala-Partio K, Laitinen O, Partio EK, et al. Comparison of the fixation of subcapital femoral neck osteotomies with absorbable self-reinforced poly-L-lactide lag-screws or metallic screws in sheep. J Orthop Res. 1997;15(1):124-127.

18. Peltoniemi HH, Hallikainen D, Toivonen T, Helevirta P, Waris T. SR-PLLA and SR-PGA miniscrews: biodegradation and tissue reactions in the calvarium and dura mater. J Craniomaxillofac Surg. 1999; 27(1):42-50.

19. Burns AE, Varin J. Poly-L-lactic acid rod fixation results in foot surgery. $J$ Foot Ankle Surg. 1998;37(1):37-41.

20. Li H, Gong M, Yang A, et al. Degradable biocomposite of nano calciumdeficient hydroxyapatite-multi(amino acid) copolymer. Int J Nanomed. 2012;7:1287-1295.

21. Dai Z, Li Y, Lu W, et al. In vivo biocompatibility of new nano-calciumdeficient hydroxyapatite/poly-amino acid complex biomaterials. Int $J$ Nanomed. 2015;10:6303-6316.

22. Li H, Yang L, Dong X, Gu Y, Lv G, Yan Y. Composite scaffolds of nano calcium deficient hydroxyapatite/multi-(amino acid) copolymer for bone tissue regeneration. J Mater Sci Mater Med. 2014;25(5):1257-1265.

23. Verheyen CC, de Wijn JR, van Blitterswijk CA, de Groot K. Evaluation of hydroxyapatite/poly(L-lactide) composites: mechanical behavior. J Biomed Mater Res. 1992;26(10):1277-1296.

24. Yao Q, Ye J, Xu Q, Mo A, Gong P. Composite scaffolds of dicalcium phosphate anhydrate/multi-(amino acid) copolymer: in vitro degradability and osteoblast biocompatibility. J Biomater Sci Polym Ed. 2015; 26(4):211-223.

25. Wu ZH. Asepsis. In: Wu ZD, Wu ZH, editors. Surgery. Beijing: People's Medical Publishing House Co., Ltd.; 2008:7-11.

26. Lane JM, Sandhu HS. Current approaches to experimental bone grafting. Orthop Clin North Am. 1987;18(2):213-225.

27. Wang M, Liu C, Xiao W. Intra-articular injection of hyaluronic acid for the reduction in joint adhesion formation in a rabbit model of knee injury. Knee Surg Sports Traumatol Arthrosc. 2014;22(7):1536-1540.

28. Proffen BL, McElfresh M, Fleming BC, Murray MM. A comparative anatomical study of the human knee and six animal species. Knee. 2012;19(4):493-499.

29. van den Borne MP, Raijmakers NJ, Vanlauwe J, et al. International Cartilage Repair Society (ICRS) and Oswestry macroscopic cartilage evaluation scores validated for use in autologous chondrocyte implantation (ACI) and microfracture. Osteoarthritis Cartilage. 2007; 15(12):1397-1402.

30. Han $\mathrm{P}$, Cheng $\mathrm{P}$, Zhang $\mathrm{S}$, et al. In vitro and in vivo studies on the degradation of high-purity $\mathrm{Mg}$ (99.99wt.\%) screw with femoral intracondylar fractured rabbit model. Biomaterials. 2015;64:57-69.

31. Shih KS, Hou SM, Lin SC. Theoretical prediction of pullout strengths for dental and orthopaedic screws with conical profile and buttress threads. Comput Methods Programs Biomed. 2017;152:159-164.

32. Hu B, Wang W, Wang Y, et al. Degradation of glutamate-based organogels for biodegradable implants: in vitro study and in vivo observation. Mater Sci Eng C Mater Biol Appl. 2018;82:80-90.

33. Seyednejad H, Gawlitta D, Kuiper RV, et al. In vivo biocompatibility and biodegradation of 3D-printed porous scaffolds based on a hydroxylfunctionalized poly(ع-caprolactone). Biomaterials. 2012;33(17): 4309-4318. 
34. Cheng $\mathrm{P}$, Han $\mathrm{P}$, Zhao $\mathrm{C}$, et al. High-purity magnesium interference screws promote fibrocartilaginous enthesis regeneration in the anterior cruciate ligament reconstruction rabbit model via accumulation of BMP-2 and VEGF. Biomaterials. 2016;81:14-26.

35. Fujii T, Noda M, Yamakawa T, Doita M. Percutaneous reduction of a displaced femoral intercondylar fracture assisted with arthroscopy and fluoroscopy. J Trauma. 2008;64(3):834-837.

36. Hayes JS, Richards RG. Surfaces to control tissue adhesion for osteosynthesis with metal implants: in vitro and in vivo studies to bring solutions to the patient. Expert Rev Med Devices. 2010;7(1):131-142.

37. Böstman O, Pihlajamäki H. Clinical biocompatibility of biodegradable orthopaedic implants for internal fixation: a review. Biomaterials. 2000; 21(24):2615-2621.

38. Sidambe AT. Biocompatibility of advanced manufactured titanium implants-a review. Materials. 2014;7(12):8168-8188.

39. Williams DF. On the mechanisms of biocompatibility. Biomaterials. 2008;29(20):2941-2953.

40. Siddharthan A, Seshadri SK, Sampath Kumar TS. Rapid synthesis of calcium deficient hydroxyapatite nanoparticles by microwave irradiation. Trends Biomater Artif Organs. 2005;18(2):110-113.

41. Guo H, Su J, Wei J, Kong H, Liu C. Biocompatibility and osteogenicity of degradable Ca-deficient hydroxyapatite scaffolds from calcium phosphate cement for bone tissue engineering. Acta Biomater. 2009;5(1):268-278.

42. Li H, Yan Y, Wei J, et al. Bone substitute biomedical material of multi(amino acid) copolymer: in vitro degradation and biocompatibility. J Mater Sci Mater Med. 2011;22(11):2555-2563.

43. Duan H, Yang H, Xiong Y, et al. Effects of mechanical loading on the degradability and mechanical properties of the nanocalciumdeficient hydroxyapatite-multi(amino acid) copolymer composite membrane tube for guided bone regeneration. Int J Nanomed. 2013;8: 2801-2807.

44. Cipriano AF, Lin J, Lin A, et al. Degradation of bioresorbable Mg-4$\mathrm{Zn}-1 \mathrm{Sr}$ intramedullary pins and associated biological responses in vitro and in vivo. ACS Appl Mater Interfaces. 2017;9(51):44332-44355.

45. Ma Z, Wu Y, Wang J, Liu C. In vitro and in vivo degradation behavior of poly(trimethylene carbonate-co-d,l-lactic acid) copolymer. Regen Biomater. 2017;4(4):207-213.

46. Han J, Wu LP, Liu XB, et al. Biodegradation and biocompatibility of haloarchaea-produced poly(3-hydroxybutyrate-co-3-hydroxyvalerate) copolymers. Biomaterials. 2017;139:172-186.

47. Nherera L, Trueman P, Horner A, Watson T, Johnstone AJ. Comparison of a twin interlocking derotation and compression screw cephalomedullary nail (InterTAN) with a single screw derotation cephalomedullary nail (proximal femoral nail antirotation): a systematic review and meta-analysis for intertrochanteric fractures. J Orthop Surg Res. 2018; 13(1):46-55.
48. Loukachov VV, Birnie MFN, Dingemans SA, de Jong VM, Schepers T. Percutaneous intramedullary screw fixation of distal fibula fractures: a case series and systematic review. J Foot Ankle Surg. 2017;56(5): 1081-1086.

49. Mudgal CS, Jupiter JB. Plate and screw design in fractures of the hand and wrist. Clin Orthop Relat Res. 2006;445:68-80.

50. Chapman JR, Harrington RM, Lee KM, et al. Factors affecting the pullout strength of cancellous bone screws. J Biomech Eng. 1996;118(3): 391-398.

51. Christodoulou E, Chinthakunta S, Reddy D, et al. Axial pullout strength comparison of different screw designs: fenestrated screw, dual outer diameter screw and standard pedicle screw. Scoliosis. 2015;10: 15-21.

52. Manon J, Hussain MM, Harris J, et al. Biomechanical investigation of a novel revision device in an osteoporotic model: pullout strength of pedicle screw anchor versus larger screw diameter. Clin Spine Surg. 2017; 30(6):265-271.

53. Innocenti M, Mancini M, Faccio M, et al. The use of a high-tech knee pad for reduction of the postoperative effusion after total knee arthroplasty. Joints. 2017;5(1):7-11.

54. Whitchelo T, McClelland JA, Webster KE. Factors associated with stair climbing ability in patients with knee osteoarthritis and knee arthroplasty: a systematic review. Disabil Rehabil. 2014;36(13):1051-1060.

55. Ha CW, Park YB, Song YS, Kim JH, Park YG. Increased range of motion is important for functional outcome and satisfaction after total knee arthroplasty in Asian patients. J Arthroplasty. 2016;31(6): 1199-1203.

56. Scarcella JB, Cohn BT. The effect of cold therapy on the postoperative course of total hip and knee arthroplasty patients. Am J Orthop. 1995; 24(11):847-852.

57. Boniforti F, Macaione A, Gagliardi S, Giangrasso F, di Marzo D, Giacco F. Early assessment of patient perception of outcome in total knee replacement. Joints. 2014;2(2):71-75.

58. Alves CJ, Neto E, Sousa DM, et al. Fracture pain-traveling unknown pathways. Bone. 2016;85:107-114.

59. Butts SC, Floyd E, Lai E, Rosenfeld RM, Doerr T. Reporting of postoperative pain management protocols in randomized clinical trials of mandibular fracture repair: a systematic review. JAMA Facial Plast Surg. 2015;17(6):440-448
International Journal of Nanomedicine

\section{Publish your work in this journal}

The International Journal of Nanomedicine is an international, peerreviewed journal focusing on the application of nanotechnology in diagnostics, therapeutics, and drug delivery systems throughout the biomedical field. This journal is indexed on PubMed Central, MedLine, CAS, SciSearch ${ }^{\circledR}$, Current Contents ${ }^{\circledR} /$ Clinical Medicine,

\section{Dovepress}

Journal Citation Reports/Science Edition, EMBase, Scopus and the Elsevier Bibliographic databases. The manuscript management system is completely online and includes a very quick and fair peer-review system, which is all easy to use. Visit http://www.dovepress.com/ testimonials.php to read real quotes from published authors. 